CASE REPORT

\title{
Erdheim-Chester disease: pulmonary infiltration responding to cyclophosphamide and prednisolone
}

\section{S C Bourke, A G Nicholson, G J Gibson}

Erdheim-Chester disease is a rare multisystem histiocytic disorder in which death most commonly results from pulmonary or cardiac involvement. We report the case history of a 55 year old man with this condition with extensive and progressive pulmonary disease. He had no response to prednisolone alone, but treatment with prednisolone plus cyclophosphamide was associated with a rapid improvement in symptoms, lung function, and the chest radiographic appearance. He subsequently showed a symptomatic, functional, and radiological deterioration, followed by a marked slowing in the rate of decline. He currently remains stable 41 months after treatment was initiated. This is the first report of pulmonary Erdheim-Chester disease showing improvement in both lung function and symptoms with any form of treatment.

$\mathrm{E}$ rdheim-Chester disease. (ECD) is a rare multisystem disease characterised by infiltration of affected tissues by foamy histiocytes, similar to but distinct from Langerhans' cell histiocytosis. The most common presentation is bone pain, followed by diabetes insipidus, exophthalmos, a retroperitoneal mass, and pulmonary or cardiac involvement. ${ }^{1}$ Symmetrical osteosclerotic lesions in the long bones, sparing the epiphyses, are virtually pathognomonic. ${ }^{1-3}$ Thoracic computed tomographic (CT) appearances include reticular and centrilobular nodular opacities, scattered ground glass shadowing, and interlobular septal and pleural thickening, often with an upper zone predominance. Adenopathy is uncommon. ${ }^{3}{ }^{4}$ Survival is determined by extraosseous disease, particularly pulmonary or cardiac involvement. ${ }^{1}$ We report a patient with ECD with advanced pulmonary disease unresponsive to steroids alone, but in whom combination therapy with cyclophosphamide and prednisolone improved symptoms, the chest radiographic appearance, and respiratory function. To our knowledge, this is the first report of any treatment producing an improvement in both dyspnoea and lung function in pulmonary ECD.

\section{CASE HISTORY}

A 55 year old man presented with a 1 year history of progressive exertional dyspnoea (1 mile), weight loss of $20 \mathrm{~kg}$, and pain in his right shoulder. He had a history of asbestos exposure (lagging pipes for $>20$ years) and was a life long non-smoker. Respiratory examination showed reduced chest expansion only. Chest radiography showed extensive bilateral upper and mid zone nodular shadowing and minor blunting of the costophrenic angles. Both humeri showed focal dense osteosclerosis (fig lA). The CT scan showed gross abnormal interstitial and some alveolar shadowing in both lung fields, particularly anteriorly in the upper and mid zones, and focal pleural thickening. No lymphadenopathy was present.
Lung function tests showed a moderate restrictive ventilatory defect (vital capacity (VC) $=81.5 \%$, total lung capacity $($ TLC) $=69.3 \%$ predicted). Serum alkaline phosphatase, angiotensin converting enzyme, immunoglobulins, autoantibody screen, avian precipitin, and prostate specific antigen levels were normal. Sputum, bronchoalveolar lavage fluid, and early morning urine were smear and culture negative for mycobacteria. A transbronchial biopsy specimen showed non-specific chronic inflammation and fibrosis; histiocytes, including multinucleate forms, were present, but no granulomas, caseation, or acid fast bacilli were identified. Immunoperoxidase stain for S100 protein was negative.

After 5 months clinical observation with serial radiography and lung function, he complained of increasing dyspnoea. Prednisolone $30 \mathrm{mg} /$ day was commenced, but after 1 month there was no symptomatic, functional, or radiographic improvement and treatment was withdrawn over 3 weeks. He subsequently became more breathless (100-200 m) with a rapid decline in lung function (fig 2) and no clinical evidence of infection. In view of the diagnostic uncertainty and rapid deterioration, a video assisted thoracoscopic lung biopsy was performed. Histological examination showed thickening of the visceral pleura and bronchovascular bundles by hyaline fibrosis. Histiocytes with abundant pale eosinophilic or foamy cytoplasm plus lymphocytes and eosinophils were present but did not aggregate into discrete granulomas. The histiocytes stained for CD68 and weakly for S100, but were negative for CDla. This immunophenotype combined with the morphological features was highly suggestive of Erdheim-Chester disease. Review of previous osteoarticular imaging showed classical changes of the disorder. ${ }^{1-3}$

In view of the symptomatic and functional deterioration, treatment was restarted with prednisolone $40 \mathrm{mg}$ plus cyclophosphamide $100 \mathrm{mg}$ daily, with mesna and oral bisphosphonate prophylaxis. Over the following 6 weeks VC and TLCo improved from 2.1 to $2.8 \mathrm{l}$, and from 3.04 to $4.02 \mathrm{mmol} / \mathrm{kPa} / \mathrm{min}$, respectively (fig 2 ), with a modest improvement in exertional dyspnoea, energy, appetite and weight. Subsequently there was a symptomatic, functional, and radiographic deterioration, followed by relative stability maintained for over 41 months.

\section{DISCUSSION}

Erdheim-Chester disease was originally described in $1930 .^{5}$ Foamy histiocytes and mononuclear cells infiltrate affected tissues, but well formed granulomas are not seen. The histiocytes may stain for S100 but not for CDla and do not show Birbeck granules on electron microscopy, distinguishing ECD from Langerhans' cell granulomatosis. The peak incidence is in the sixth decade. Pulmonary ECD typically presents with dyspnoea and sometimes a dry cough. ${ }^{23}$ In the largest review of reported cases, 22/37 patients died during follow up, eight within 6 months of diagnosis. ${ }^{1}$ Treatment on empirical grounds has been attempted with steroids, 

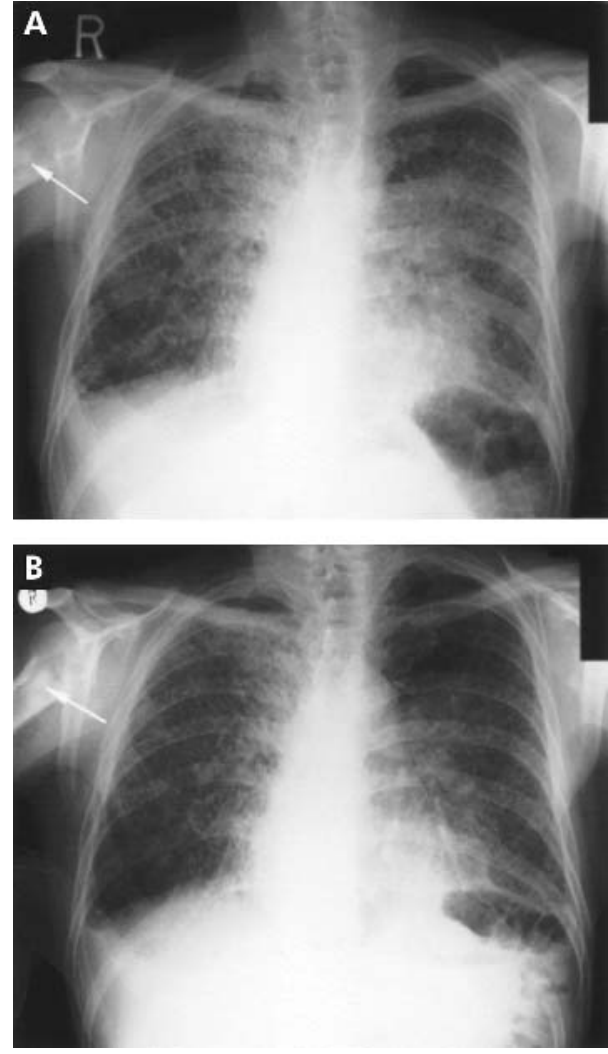

Figure 1 Chest radiograph $(A)$ before treatment with cyclophosphamide and prednisolone showing extensive nodular shadowing in the lung fields, pleural thickening in the costophrenic angles, and osteosclerosis (arrow) with sparing of the epiphyses and (B) after 2 months of treatment with cyclophosphamide and prednisolone showing substantial clearing of the nodular shadowing in the lung fields.

cyclophosphamide, vinblastine, vincristine, adriamycin, colchicine, and radiotherapy in varying combinations, with a clinical response in only a minority of cases. ${ }^{1-3}$ Steroid therapy has been reported to improve exophthalmos, ${ }^{67}$ renal and bone involvement ${ }^{7}$ and, in combination with vinblastine plus adriamycin or cyclophosphamide, exophthalmos, renal and retroperitoneal disease. ${ }^{8}$ Radiotherapy transiently relieves bone pain but not exophthalmos. Improvement in respiratory symptoms has been described with steroids alone $^{29}$ and in combination with cyclophosphamide and etoposide, ${ }^{3}$ but no previous reports have shown objective improvement. Sustained improvement and stabilisation of disease is unusual.

In our patient the radiographic appearance at presentation was considered suggestive of sarcoidosis, but the initial transbronchial biopsy was inconclusive and the bone changes, absence of lymphadenopathy, and lack of response to corticosteroids made sarcoidosis unlikely. The correct diagnosis was made only after video assisted thoracoscopic biopsy.

Our patient was followed for several months before the initial trial of prednisolone, establishing a baseline rate of decline. Although he showed no response to prednisolone alone, the disease appeared to take a much more aggressive course following the withdrawal of steroid treatment. The significance of this observation is uncertain. The natural

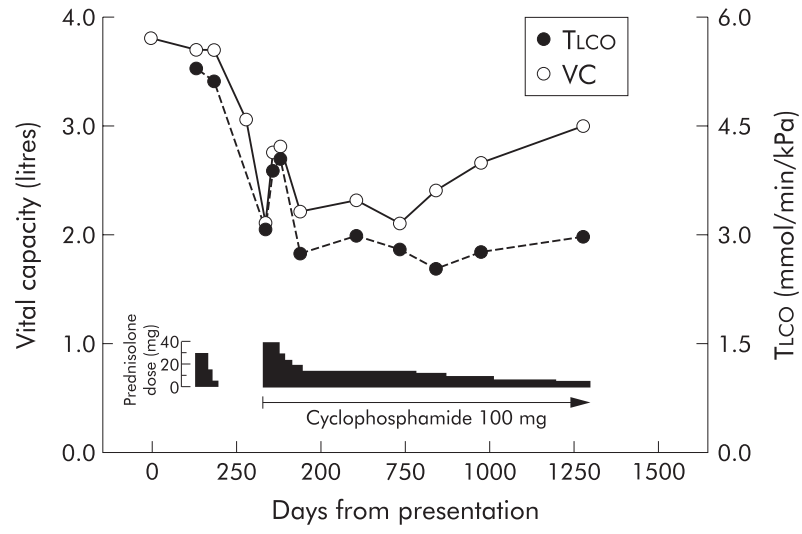

Figure 2 Rapid decline in vital capacity (VC) and transfer factor (TLCO) after the initial trial of prednisolone was discontinued, which subsequently improved and stabilised following initiation of treatment with cyclophosphamide and prednisolone.

history of pulmonary ECD is unpredictable, but combined treatment with prednisolone and cylclophosphamide in this case appears initially to have improved symptoms, function and radiology, and subsequently slowed the rate of disease progression (fig 2), suggesting that this may be a useful treatment for an otherwise progressive pulmonary condition. A period of observation before initiating treatment is useful to gauge the rate of decline, particularly in unusual conditions and when novel treatment is being considered. The low incidence of ECD and consequent lack of prospective controlled studies, at present or within the foreseeable future, presents difficulties in making therapeutic decisions.

\section{Authors' affiliations}

S C Bourke, Consultant Respiratory Physician, Northumbria Healthcare Trust and Freeman Hospital, Newcastle upon Tyne, UK

A G Nicholson, Department of Histopathology, Royal Brompton Hospital, London, UK

G J Gibson, Professor of Respiratory Medicine, University of Newcastle and Freeman Hospital, Newcastle upon Tyne NE7 7DN, UK

Correspondence to: Dr S C Bourke, North Tyneside General Hospital, Rake Lane, North Shields NE29 8NH, UK; sbourke@doctors.org.uk

Accepted for publication 24 June 2002

\section{REFERENCES}

1 Veyssier-Belot C, Cacoub P, Caparros-Lefebvre D, et al. Erdheim-Chester disease. Clinical and radiologic characteristics of 59 cases. Medicine 1996;75: 157-69.

2 Rush WL, Andriko JA, Galateau-Salle F, et al. Pulmonary pathology of Erdheim-Chester disease. Mod Pathol 2000;13:747-54.

3 Egan AJ, Boardman LA, Tazelaar HD, et al. Erdheim-Chester disease: clinical, radiologic, and histopathologic findings in five patients with interstitial lung disease. Am J Surg Pathol 1999;23:17-26.

4 Wittenberg KH, Swensen SJ, Myers JL. Pulmonary involvement with ErdheimChester disease: radiographic and CT findings. AJR 2000;174:1327-31.

5 Chester W. Uber lipoidgranulomatose. Virchows Arch Pathol Anat 1930;279:561-602.

6 Valmaggia C, Neuweiler J, Fretz C, et al. A case of Erdheim-Chester disease with orbital involvement. Arch Ophthalmol 1997;115:1467-8.

7 Bellin M, Cacoub P, Wechsler J, et al. Erdheim-Chester disease associated with renal involvement and thrombocythemia. Eur J Radiol 1993;3:266-9.

8 Rozenberg I, Wechsler J, Koenig F, et al. Erdheim-Chester disease presenting as malignant exophthalmos. Br J Radiol 1986;59:173-7.

9 Capparros-Lefebvre D, Pruvo J, Remy M, et al. Neuroradiological aspects of Erdheim-Chester disease. Am J Neuroradiol 1995;16:735-40. 